previously reported ${ }^{1}$. Our patients showed low levels of SLEDAI/SLICC. There was not relation between activity levels and baseline damage with the presence of depression ${ }^{4}$. FACIT IV scale was a good independent predictor of fatigue in SLE patients with or without depression vs controls.

References:

[1] L Palagini M et al Depression and systemic lupus erythematosus: a systematic review. Lupus 2013

[2] Hugo FJ et al DSM-III-R classification of psychiatric symptoms in systemic lupus erythematosus. Psychosomatics 1996.

[3] Lai JS et al Validation of the Functional Assessment of Chronic Illness TherapyFatigue Scale in Patients with Moderately to Severely Active Systemic Lupus Erythematosus, Participating in a Clinical Trial Journal of Rheumatology 2011.

[4] Segui J et al Psychiatric and psychosocial disorders in patients with systemic lupus erythematosus: A longitudinal study of active and inactive stages of the disease. Lupus 2000.

Disclosure of Interest: None declared

DOI: 10.1136/annrheumdis-2017-eular.6198

\section{FRI0277 ANTI-SSA ANTIBODY STATUS IN COMBINATION WITH ULTRASOUND OF MAJOR SALIVARY GLANDS: A SHORTCUT IN THE CLASSIFICATION OF PRIMARY SJÖGREN'S SYNDROME?}

E. Mossel $^{1}$, K. Delli ${ }^{2}$, J.F. Van Nimwegen ${ }^{1}$, A.J. Stel ${ }^{1}$, F.G.M. Kroese ${ }^{1}$ F.K.L. Spijkervet ${ }^{2}$, A. Vissink ${ }^{2}$, S. Arends ${ }^{1}$, H. Bootsma ${ }^{1}$ on behalf of EULAR US-pSS Study Group. ${ }^{1}$ Rheumatology and Clinical Immunology; ${ }^{2}$ Oral and Maxillofacial Surgery, University Medical Center Groningen, Groningen, Netherlands

Background: Ultrasound of major salivary glands (sUS) is an upcoming diagnostic method to assess the involvement of major salivary glands in primary Sjögren's syndrome (pSS). In the AECG, ACR and recently published ACR-EULAR criteria, a positive salivary gland biopsy and/or presence of anti-SSA antibodies are necessary to classify a patient as pSS, while sUS is not included as a diagnostic item. Objectives: To assess whether combining anti-SSA antibody status with sUS outcome can predict classification of patients as pSS in our inception cohort study.

Methods: Consecutive outpatients clinically suspected with pSS underwent sUS of the parotid and submandibular glands. Parenchymal echogenicity, homogeneity, hypoechogenic areas, hyperechogenic reflections and clearness of salivary gland border were scored according to the Hocevar scoring system (total score $0-48){ }^{1}$ Positive sUS was defined as total score $\geq 15$. Patients underwent a diagnostic work up according to the AECG, ACR and ACR-EULAR criteria. We analyzed the predictive value of the combination of anti-SSA antibody status and sUS outcome for classification as pSS or non-pSS. Separate analyses were done considering either i) parotid gland biopsy or ii) labial gland biopsy as an item, when applying these classification criteria.

Results: Anti-SSA antibody status was positive in $53(51 \%)$ patients and sUS was positive in $40(39 \%)$ patients.

When parotid gland biopsy outcome was considered as an item of the criteria, 45 of 97 patients were classified as pSS according to the AECG, 44 of 97 according to the ACR and 52 of 99 according to the ACR-EULAR criteria. The combination of presence of anti-SSA antibodies with positive sUS showed a very high positive predictive value for classification as pSS (94-97\%) and the combination of absence of anti-SSA antibodies with negative sUS highly excludes classification (negative predictive value $98-100 \%$ ).

When labial gland biopsy outcome was considered as an item of the criteria, 49 of 96 patients were classified as pSS according to the AECG, 43 of 93 according to the ACR and 55 of 97 according to the ACR-EULAR criteria. The combination of presence of anti-SSA antibodies with positive sUS showed a high positive predictive value for classification as pSS (94-97\%). However, the combination of absence of anti-SSA antibodies with negative sUS did not per se exclude classification (negative predictive value $89-93 \%$ ).

Conclusions: In our prospective inception cohort study derived from daily clinical practice, the combination of presence of anti-SSA antibodies and positive sUS outcome highly predicts classification as pSS according to the AECG, ACR and ACR-EULAR classification criteria.

References:

[1] Hocevar et al. Rheumatology (Oxford) 2005;44:768-72.

Disclosure of Interest: None declared

DOI: 10.1136/annrheumdis-2017-eular.5938

\section{FRI0278 PREVALENCE OF PRIMARY SJÖGREN'S SYNDROME IN A POPULATION-BASED COHORT IN THE UNITED STATES}

G. Maciel ${ }^{1,2}$, C. Crowson ${ }^{3}$, E. Matteson ${ }^{2}$, D. Cornec $4,5 .{ }^{1}$ Maciel Hospital, Montevideo, Uruguay; ${ }^{2}$ Rheumatology; ${ }^{3}$ Division of Biomedical Statistics and Informatics, Department of Health Sciences Research, Mayo Clinic, Rochester Minnesota, United States; ${ }^{4}$ Rheumatology, CHRU Brest, Brest, France; ${ }^{5}$ Pulmonary and Critical Care Medicine, Mayo Clinic, Rochester Minnesota, United States

Background: Different studies worldwide reported highly discrepant estimates for the prevalence of primary Sjögren's syndrome (pSS), ranging from $0.01 \%$ of the general population to more than $3 \%$. No previous study reported the prevalence of pSS in the United States.

Objectives: To report the 2015 point prevalence of pSS in the first populationbased study performed in the U.S

Methods: Cases of all potential pSS patients living in Olmsted County, Minnesota on January 1, 2015 were retrieved using the Rochester Epidemiology Project resources, and ascertained by manual medical record review. Definite pSS cases were defined according to physician diagnosis. All patients with doubtful cases and all patients with an associated systemic autoimmune disease were excluded. The use of diagnostic tests was assessed and the performance of classification criteria in this community-based cohort was evaluated. The number of prevalent cases in 2015 was also projected based on 1976-2005 incidence data from the same source population.

Results: A total of 106 patients with pSS were included in the study: $86 \%$ were female, with a mean (SD) age of 64.6 (15.2) years and disease duration of 10.5 (8.4) years. A majority were anti-SSA positive (75\%) and/or anti-SSB positive (58\%), but only $22 \%$ met American-European Consensus Group or American College of Rheumatology criteria because the other tests required for disease classification were rarely performed in clinical practice (ocular dryness objective assessment, salivary gland functional or morphologic tests, or salivary gland biopsy). According to the physician diagnosis, age and sex adjusted prevalence of pSS was $10.3 / 10,000$ inhabitants, but according to classification criteria this prevalence would be only $2.2 / 10,000$. The analysis based on previous incidence data projected a similar 2015 prevalence rate of $11.0 / 10,000$. Using figures from the 2015 general U.S. population census, a total of 248,000 patients with pSS (35,000 males and 213,000 females) would currently live in the country. If only cases fulfilling classification criteria are considered, there would be only about 53,000 prevalent cases of pSS in the U.S.

Conclusions: This study reports the first prevalence rate of physician-diagnosed pSS in a well-defined population in the U.S. The estimated prevalence of $10.3 / 10,000$ inhabitants in 2015 is higher than previous results obtained in other geographical areas, probably due to different methodological designs of the studies. Because physicians rarely used tests included in the classification criteria to diagnose the disease in this community setting, current classification criteria do not reflect accurately the diagnosis of pSS in routine clinical practice.

Disclosure of Interest: None declared

DOI: 10.1136/annrheumdis-2017-eular.1179

\section{FRI0279 PREVALENCE AND FACTORS ASSOCIATED WITH PRECONCEPTIONAL COUNSELING IN WOMEN WITH AUTOIMMUNE RHEUMATIC DISEASES}

G. García-Garza ${ }^{1}$, M.A. Saavedra ${ }^{1}$, D. Miranda-Hernández ${ }^{1}$, A. Sánchez ${ }^{1}$, G. García-Collinot ${ }^{2}$, R. Bustamante-González ${ }^{1}$, L.J. Jara ${ }^{3} .{ }^{1}$ Rheumatology; ${ }^{2}$ Research Division; ${ }^{3}$ Education and Research Direction, Hospital de Especialidades, Cmn la Raza, IMSS, México, Mexico

Background: Adequate prenatal counseling in women with autoimmune rheumatic diseases (ARDs) may help minimize maternal-fetal complications. However, the available information about the frequency and quality of prenatal counseling given to these patients is limited.

Objectives: To estimate the prevalence of preconceptional counseling and associated factors in women with ARDs.

Methods: A survey was conducted on socio-demographic data, gynecologicalobstetric history, and domains related to preconception counseling in women with ARDs at the reproductive stage. It was defined as adequate preconception counseling if the patient knew that the pregnancy should be medically planned, that the complications are associated with severity/activity of the disease, that they should use an effective contraceptive method and that their medications may or may not be used during pregnancy. Descriptive statistics, Student's $T$ test for quantitative variables with normal distribution, Mann-Whitney $U$ test for non-normal and chi-square distribution were used for categorical or ordinal variables.

Results: Of a total of 146 surveys, 131 were analyzed. Only $49(37.4 \%)$ patients received adequate preconception counseling. Two thirds of the patients had systemic lupus erythematosus (Table). The time of evolution of the disease was a factor associated with receiving adequate counseling (5.6 vs 3.6 years, $p=0.023$ ). Illness, marital status, and level of education were not factors associated with

\begin{tabular}{|c|c|c|c|}
\hline & $\begin{array}{l}\text { Adequate Counseling } \\
\qquad(\mathrm{n}=49)\end{array}$ & $\begin{array}{l}\text { Inadequate counseling } \\
(\mathrm{n}=82)\end{array}$ & $P$ value \\
\hline Age (years) ${ }^{a}$ & $30.8 \pm 7.0$ & $30.5 \pm 8.5$ & 0.836 \\
\hline Time of evolution (years) ${ }^{b}$ & $5.6(0.25-29)$ & $3.6(0.25-27)$ & 0.023 \\
\hline Previous pregnancies ${ }^{b}$ & $1(0-4)$ & $1(0-5)$ & 0.192 \\
\hline Rheumatoid arthritis ${ }^{c}$ & $13(26.5 \%)$ & $18(22 \%)$ & 0.69 \\
\hline Systemic lupus erythematosus ${ }^{c}$ & $31(63.3 \%)$ & $52(63.4 \%)$ & \\
\hline Use of contraceptive method ${ }^{c}$ & $31(63.3 \%)$ & $44(53.7 \%)$ & 0.282 \\
\hline Use of embryotoxic/teratogenic drugs ${ }^{c}$ & $39(79.6 \%)$ & $34(41.5 \%)$ & 0.001 \\
\hline Counseling for a rheumatologist ${ }^{\mathrm{C}}$ & $37(75.5 \%)$ & $28(34.1 \%)$ & 0.001 \\
\hline Counseling by other doctors ${ }^{c}$ & $35(24.5 \%)$ & $31(65.9 \%)$ & 0.197 \\
\hline Professional education ${ }^{c}$ & $23(46.9 \%)$ & $31(37.8 \%)$ & 0.616 \\
\hline Married $^{c}$ & $15(30.6 \%)$ & $32(39 \%)$ & 0.774 \\
\hline
\end{tabular}

a Student's t-test; ${ }^{\text {} U}$ of Mann Whitney; ${ }^{\mathrm{c}} \mathrm{Chi}$ square. 
adequate counseling. The adoption of a contraceptive method and the number of pregnancies were not different between the two groups. The history of use of embryotoxic/teratogenic drugs was associated with a higher frequency of adequate counseling $(79.6 \%$ vs $41.5 \%, p=0.001)$. According to the patients, adequate counseling was given by the rheumatologist in $75.5 \%$ of the cases $(p=0.001)$. Conclusions: Adequate preconceptional counseling in our patients with ARDs at the reproductive stage is deficient. A multidisciplinary strategy is required to improve the frequency and quality of preconception counseling in patients with ARDs.

Disclosure of Interest: None declared

DOI: 10.1136/annrheumdis-2017-eular.6475

\section{FRI0280 ASSOCIATION BETWEEN INSULIN RESISTANCE, SUBCLINICAL ATHEROSCLEROSIS AND ACTIVITY/DAMAGE STATUS IN SLE PATIENTS}

H. Sánchez-Pérez ${ }^{1}$, B. Tejera Segura ${ }^{1}$, M.A. Gonzalez-Gay ${ }^{2}$, I. Ferraz-Amaro ${ }^{1}$ ${ }^{1}$ Rheumatology, Hospital Universitario de Canarias, la Laguna; ${ }^{2}$ Rheumatology, Hospital Marques de Valdecilla, Santander, Spain

Background: Insulin resistance (IR) may contribute to an increase in cardiovascular risk in general population as well as in Systemic Lupus Erythematosus (SLE) patients.

Objectives: The aim of this study was to examine the association between IR and disease activity, disease characteristics, drug exposure and subclinical atherosclerosis in patients with SLE.

Methods: Cross-sectional study that encompassed 87 SLE patients and 82 sex-matched controls. IR by homeostatic model assessment (HOMA2), insulin, $\mathrm{C}$-peptide serum levels and lipid profile were assessed in both groups. Activity (SLEDAI), severity (Katz) and damage (SLICC) scores, carotid intima-media thickness (cIMT) and carotid plaques (ultrasound) were assessed in SLE patients. A multivariable regression analysis, adjusted for IR related factors, was performed to evaluate the differences between groups in IR indexes and, in SLE patients, the interrelation between IR and disease activity/characteristics as well as subclinical atherosclerosis.

Results: Median disease duration was 16 years (IQR 9-21). Body mass index, abdominal circumference, hypertension or dyslipidemia did not differ between groups. According to the SLEDAI score, $40 \%$ of patients were in no activity, while $32,21,18$ and $1 \%$ were in mild, moderate, high and very high activity respectively. HOMA-IR-C-peptide (beta coefficiente 0.53 , [95\% Cl 0.25-0.82], $p=0.00$ ) was increased in SLE patients when compared to controls, as well as HOMA \%B C peptide levels (beta coef. $35,95 \% \mathrm{Cl} 18-52, p=0.00$ ). Similarly, insulin sensitivity estimated through HOMA-S\% was inferior in SLE patients (-beta coef. - $37,95 \%$ $\mathrm{Cl}-63-11, \mathrm{p}=0.01)$. This diferences remained significant even after adjustment for IR related factors.

SLICC damage index was clearly asociated with IR indexes; higher index values were related to higher HOMA-IR-C-peptide (beta coef. 37, [95\% Cl 16-57], $p=0.00$ ) and lower HOMA-S\% levels (beta coef. 30\%, [95\% Cl -47-14], p=0.00). Katz severity index showed correlation with HOMA-IR-C-peptide (beta coef.-5, $[95 \% \mathrm{Cl}-11-0], \mathrm{p}=0.04)$. These associations remained significant after adjustment for age, gender, smoking, hypertension and dyslipidemia, and, in relation with the SLICC index, also after adjustment for prednisone intake. SLEDAI activity index was not related to IR indexes.

The use of prednisone was positively associated with HOMA-IR both when considered binary (beta coef $47,[95 \% \mathrm{Cl} 31-63], \mathrm{p}=0.00$ ) and continous (beta coef 2 [95\% Cl 0-5] per mg, $p=0.03$ ). Hydroxycloroquine (or other drugs) use was not associated with IR indexes, neither were disease duration, antiDNA titers and complement serum levels.

Carotid plaques were found in $20 \%$ of the SLE patients. The presence of carotid plaques was correlated with a higher HOMA-IR-C-peptide (OR 3.15 [95\% IC 1.17-8.51], $\mathrm{p}=0.02)$, and a higher cIMT value was associated with a lower HOMAIR-S\%>C-peptide (beta coef. 0.98 [95\% Cl 0.96-0.99], $p=0.03$ ). Nevertheless, after adjustment for cardiovascular risk factors this relation was lost.

Conclusions: Activity and damage indexes in SLE patients are independently related to the development of IR. IR is associated with subclinical carotid atherosclerosis in SLE patients on the univariate analysis.

Disclosure of Interest: None declared

DOI: 10.1136/annrheumdis-2017-eular.2367

\section{FRI0281 UTILITY OF MORNING SAMPLE OF URINE PROTEIN/CREATININE RATIO FOR ASSESSMENT OF PROTEINURIA IN PATIENTS WITH LUPUS NEPHRITIS}

H. Zhang, J. Liang, B. Hua, L. Sun. Department of Rheumatology and Immunology, The Affiliated Drum Tower Hospital of Nanjing University Medical School, Nanjing, China

Background: Proteinuria is the principal urinary biomarker for the screening of Lupus nephritis (LN) and for monitoring disease progression. 24-hour urine collection has been the foundation for monitoring disease activity in patients with $\mathrm{LN}$, but collections are often inaccurate and inconvenient. The European League Against Rheumatism and American College of Rheumatology have recommended the urine protein/creatinine ratio (UPCR) for use in management of LN.
Objectives: We aimed to evaluate the diagnostic accuracy of the morning sample of UPCR compared with 24-hour urine collection for the detection of proteinuria and to determine the UPCR for different proteinuria ranges in patients with LN.

Methods: Three hundred and thirty seven LN patients were enrolled. The correlation between the UPCR in the morning spot urine samples and urinary protein excretion in the 24-hour collections was examined using the Pearson correlation test. The best cutoffs for UPCR predicting a 24-hour protein excretion were determined with the receiver operating characteristic curve (ROC).

Results: It was found a good positive correlation between the UPCR and 24-hour protein excretion, with a correlation coefficient ( $r$ ) of 0.891 (Fig 1). The best cutoff which gave the maximum area under the curve was 0.44 for $0.5 \mathrm{~g}, 0.92$ for $1.0 \mathrm{~g}$, 2.21 for $2.0 \mathrm{~g}, 2.70$ for $3.0 \mathrm{~g}, 3.49$ for $4.0 \mathrm{~g}$ and 4.59 for $5.0 \mathrm{~g}$.

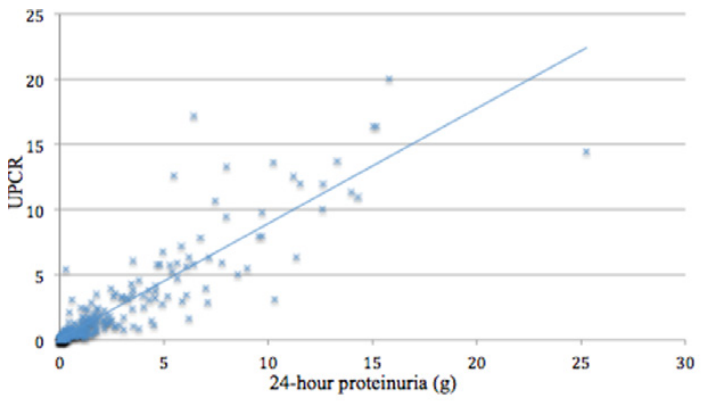

Fig. 1 Scatterplot of correlation between protein content in a 24-hour urine collection sample and morning sample of UPCR

Conclusions: The UPCR can be used as a screening test as a good predictor for proteinuria of LN patients. Also, UPCR is a valuable tool with which to monitor disease progression.

References:

[1] Bertsias GK, Tektonidou M, Amoura Z, et al. Joint European League Against Rheumatism and European Renal Association-European Dialysis and Transplant Association (EULAR/ERA-EDTA) recommendations for the management of adult and paediatric lupus nephritis. Ann Rheum Dis 2012; 71: 1771-82.

[2] Renal Disease Subcommittee of the American College of Rheumatology Ad Hoc Committee on Systemic Lupus Erythematosus Response Criteria. The American College of Rheumatology response criteria for proliferative and membranous renal disease in systemic lupus erythematosus clinical trials. Arthritis Rheum 2006; 54: 421-32.

[3] Medina-Rosas J, Yap KS, Anderson M, et al. Utility of Urinary ProteinCreatinine Ratio and Protein Content in a 24 -Hour Urine Collection in Systemic Lupus Erythematosus: A Systematic Review and Meta-Analysis. Arthritis Care Res (Hoboken) 2016; 68: 1310-9.

Disclosure of Interest: None declared

DOI: 10.1136/annrheumdis-2017-eular.5324

\section{FRI0282 CARDIOVASCULAR DAMAGE IN DECEASED PATIENTS WITH SYSTEMIC LUPUS ERYTHEMATOSUS}

I. Padjen ${ }^{1,2}$, M. Erceg ${ }^{3}$, M. Cerovec ${ }^{1,2}$, M. Mayer ${ }^{1,2}$, R. Stevanović ${ }^{3}$, B. Anić ${ }^{1,2}$ ${ }^{1}$ Department of Internal Medicine, Division of Clinical Immunology and Rheumatology, University Hospital Centre Zagreb; ${ }^{2}$ University of Zagreb, School of Medicine, ${ }^{3}$ Croatian Institute of Public Health, Zagreb, Croatia

Background: Cardiovascular comorbidities are a major contributor of damage in patients with SLE. They are driven by classical, as well as SLE-related risk factors, i.e disease activity and immunosuppressive treatment.

Objectives: We aimed to analyze cardiovascular damage (CVD) in a group of 90 deceased SLE patients regularly followed-up in a routine academic setting at our Department, and to identify features associated with accrual of CVD.

Methods: We retrospectively observed 90 SLE patients (68 females) deceased within the 2002-2011 period. All patients were $\geq 18$ years of age and Croatian residents at the time of death, fulfilling $>4$ classification criteria of the American College of Rheumatology (ACR). We identified patients with CVD, including the following components of the Systemic Lupus International Collaborating Clinics (SLICC)/ACR damage index: cardiovascular damage as defined by the index (cardiac damage), peripheral vascular damage, cerebrovascular accident, pulmonary infarction, bowel infarction and avascular necrosis. An extensive set of variables was compared between patients with and without CVD: demographics, ACR criteria at diagnosis and death, damage (according to the SLICC/ACR index) and its components one year following diagnosis and at the time of death, disease activity at diagnosis (according to the European Consensus Lupus Activity Measurements index, ECLAM), as well as features of the metabolic syndrome, smoking and immunosuppressive treatment. Frequencies were compared using the $\chi^{2}$ and Fisher's exact test, and continuous variables using the t-test and Mann-Whitney $U$ test. Variables associated with CVD in the univariate analysis were included in a multivariate logistic regression model.

Results: We identified 63/90 patients with CVD, including 46/63 (73\%) with cardiac damage, 19/63 (30\%) with peripheral vascular damage, 21/63 (33\%) 